\title{
Prostatic urethra recurrence after transurethral resection of bladder tumor (TURBT) for non-muscle-invasive bladder cancer (NMIBC)
}

\author{
Vladimir Bilim ${ }^{1}$ and Senji Hoshi ${ }^{2}$ \\ ${ }^{1}$ Kameda First Hospital \\ ${ }^{2}$ Yamagata Tokushukai Hospital
}

October 30, 2021

\begin{abstract} underappreciated. Here we present two cases having urethral recurrence after TURBT. non-muscle-invasive bladder cancer (NMIBC)

Vladimir Bilim1*, Senji Hoshi2 city, Niigata prefecture, JAPAN

Tel +81-25-382-3111, FAX +81- 25-382-7311

Authors affiliations:

1 Kameda Daiichi Hospital, Nishi-machi 2-5-22, Konan-ku, Niigata city, Japan

2 Yamagata Tokushukai Hospital, Kiyozumimachi 2-3-51, Yamagata city, Japan

Vladimir Bilim vbilim@zoho.com

Senji Hoshi senjihoshi47@yahoo.co.jp
\end{abstract}

Urinary bladder cancer is frequently multifocal and has a high incidence of recurrence. Although the prostatic urethra is a frequent site of tumor relapse in patients with non-muscle-invasive bladder cancer treated with TURBT, such tumors are often

Prostatic urethra recurrence after transurethral resection of bladder tumor (TURBT) for

*Corresponding author Vladimir Bilim, Zip code 950-0165, 2 Chome-5-22 Nishimachi, Konan Ward, Niigata

Keywords: bladder cancer, urothelial cancer, recurrence, prostatic urethra, urethra, transurethral resection of bladder tumor (TURBT)

Abbreviations:

BCG - bacillus Calmètte-Guerin

BT - bladder tumor

CIS - carcinoma in situ

LN - lymph node

NMIBC - non-muscle-invasive bladder cancer

OS - Overall survival

PU - prostatic urethra 
$\mathrm{RC}$ - radical cystectomy

TUR - transurethral resection

TURBT - transurethral resection of bladder tumor

$\mathrm{UC}$ - urothelial cancer

\section{Abstract}

Urinary bladder cancer is frequently multifocal and has a high incidence of recurrence. Although the prostatic urethra is a frequent site of tumor relapse in patients with non-muscle-invasive bladder cancer treated with TURBT, such tumors are often underappreciated. Here we present two cases having urethral recurrence after TURBT.

\section{Key Clinical Message}

Recurrence of urothelial cancer in the prostatic urethra after TURBT might be a challenge due to the microscopic involvement. Prostatic stromal invasion poses a high risk of metastasis to the pelvic LNs and requires aggressive treatment.

\section{Introduction}

Urothelial cancer (UC) is the most common malignancy of the urinary bladder. It is frequently multifocal and has a high incidence of recurrence. Bladder carcinoma in situ (CIS) especially prone to recurrence. Transurethral resection of a bladder tumor (TURBT) is a standard of care in newly diagnosed bladder tumors. Even in non-invasive tumors radically treated with TURBTs, recurrence rates are high with recurrences reaching up to $80 \%$. Recurrences can occur in the bladder and throughout all urinary tract including the urethra. Urethral recurrence after radical cystectomy (RC) without urethrectomy is frequently discussed in the literature. It is an indicator of poor survival $(4,19)$. Risk factors for urethral recurrence after RC are the presence of tumors in the trigone of the urinary bladder, multiple or recurrent(14) tumors, non-papillary bladder tumor(8), and bladder CIS.

Although the prostatic urethra (PU) is a frequent site of tumor relapse in patients with non-muscle-invasive bladder cancer (NMIBC), such tumors are often underappreciated. Risk factors for superficial urothelial cancer in the PU are high-grade, multifocal bladder UC and the presence of CIS in the bladder. Urethral involvement is diagnosed using cystoscopy and urinary cytology. However, the diagnosis might be a challenge due to the microscopic involvement of the PU. Prostatic stromal invasion has a much higher lymph node (LN) metastasis rate compared with patients without stromal invasion. Lymph drainage of tumors located in the PU occurs to the pelvic LNs. There are only sporadic reports on urethral recurrence after TURBT. Here we present two cases having urethral recurrence after TURBT.

\section{Case presentation}

Case 1. An 86-year old man was referred to our hospital with the chief complaint of asymptomatic microscopic hematuria. He was taking warfarin for atrial fibrillation and had a history of cardiogenic cerebral infarction. Cystoscopy revealed a broad base papillary tumor and area suspicious for CIS in the bladder. No urethral tumor was found. Urinary cytology was class V. The tumor was resected transurethrally. No residual tumor was detected after the TUR. At the end of the operation, intravesical instillation of $40 \mathrm{mg}$ mitomycin $\mathrm{C}$ was done. Histopathological examination revealed a non-invasive (pTa) high grade (G2) UC. Cystoscopy and CT scan revealed neither local recurrence nor distant metastases 3 months after the operation. During the follow-up cystoscopy 6 months after the initial TURBT, a recurrent bladder tumor in the bladder neck extending to the PU to the level of verumontanum was revealed and the second TURBT was performed. At the time of operation, the bladder neck tumor was resected without a residual tumor. However, resection of the PU (Fig. 1A) revealed a deep invasion into the prostate. Radical resection was impossible because of the spread of cancer to the prostate. The pathological diagnosis was UC with prostate stromal invasion (Fig.1B). Salvage EBRT was offered, but the patient and the family opted for follow-up. One month after 
the operation urethral tumor was found during cystoscopy (Fig.1C). No bladder recurrence was present at that time. CT revealed pelvic LN metastasis, obturator LN (Fig. 1D), and pararectal LN(Fig.1E) metastasis which gradually enlarged (Fig.1F) in the following CTs. There were several hemorrhage episodes from the urethral tumor which were successfully treated by placement of a urethral catheter and carbazochrome IV injections. Warfarin was changed to apixaban. The patient died a natural death at the age of 89 with a stable disease 15 months after the last TUR.

Case 2. An 80-year old man was referred to our hospital with the chief complaint of asymptomatic microscopic hematuria. Multiple papillary bladder tumors located at bladder walls and dome and also involving the trigone and bladder neck were diagnosed (Fig. 2A) and TURBT was performed. Another TURBT was performed 2 weeks later for residual bladder tumors. At the end of the operation, intravesical instillation of 40mg mitomycin C was done. The pathological diagnosis was invasive (pT1) high-grade UC. Second look TUR was performed, which revealed no residual tumor. During the follow-up cystoscopy solitary broad-based papillary tumor on the left bladder wall was found and six months after the initial TUR the fourth TURBT was performed. After the operation intravesical instillation of $30 \mathrm{mg}$ pirarubicin (THP) was done. Pathological diagnosis was non-invasive (pTa) low grade (G1) papillary UC. Ten months after the initial TUR cystoscopy revealed a papillary tumor near the verumontanum and wide transurethral resection was performed (Fig. 2B, C, D). Histopathological examination revealed a papillary UC without prostate tissue invasion (Fig. 2E). Surgical margins were negative for malignancy. Follow-up urinary cytology is negative. Cystoscopy (Fig. 2F) and CT scan revealed neither local recurrence nor metastases. The patient is disease-free 2 years from the initial diagnosis (14 months after the last TUR).

\section{Discussion}

The incidence of primary urethral UC is low. It does not exceed a few percent $(9,10)$. There is also a report on male anterior urethra $\mathrm{UC}(2)$

There are several case reports on a primary UC of the prostate(23) (22).

Primary UC of the PU is rare, occurring in as less as $1-4 \%$ of patients(1). On the other hand, the estimated incidence of bladder UC with synchronous or metachronous involvement of the prostate has been reported to be between $12 \%$ and $40 \%(3,13,18)$. In patients diagnosed with superficial bladder cancer $3.5 \%$ had macroscopic and $2.7 \%$ microscopic involvement of the PU.(16)

The prostate is a frequent site of tumor relapse in patients with superficial bladder tumors(6). Some patients may have unrecognized PU tumors at the time of primary diagnosis of bladder cancer. There is a report on a high incidence of recurrent extravesical UC after successful control of a primary bladder tumor. Twenty-four percent of men developed PU tumors. The median time to the detection of these recurrences was 11 months. PU recurrences were often diagnosed incidentally(5).

UC in PU may be either noninvasive, involving urethral mucosa and prostatic ducts, or invasive, extending to acini and stroma of the prostate. Depth of invasion is a predictor of survival. Stromal invasion of the prostate has a reported incidence between $7 \%$ and $17 \%(20)$. In one series, half of these relapses presented with prostate stroma invasion(5). Stromal invasion is associated with drastically decreased survival $(6,18)$. Prostate ductal involvement after RC poses a high risk of metastasis(21). Patients with PU involvement after RC also demonstrate various survival. Overall survival (OS) is $100 \%$ in the case of superficial tumors, and it decreases steeply to $50 \%$ and $40 \%$ in cases with ductal involvement and stromal involvement, respectively(18). Invasive tumors can present as an intraurethral or direct prostatic invasion. Contiguous growth into the prostate is also associated with a worse prognosis(12).

Higher grade, stage, multiple bladder tumors, and presence of carcinoma in situ (CIS) in the bladder were demonstrated to be risk factors for PU recurrence in patients with superficial bladder UC(16, 17). Also, tumors in the bladder trigonum were speculated to have a higher PU recurrence rate.

Although regular cystoscopy is the gold standard for diagnosis of PU recurrence in bladder cancer patients, tumors may be present in the normal-appearing urethra. In patients with persistent positive urinary cytology 
and negative cystoscopic findings, TUR biopsy at the 5 and 7 o'clock position of the verumontanum is recommended. However, low grade tumors may present with negative urinary cytology.

Superficial UC of the PU can be treated with either TUR, BCG instillations, or a combinatory approach. In non-invasive tumors, the complete response of BCG without TUR is up to $75 \%$ and 5 -yr RFS rates of 90\%. Conservative treatment options also include intravesical instillation of Mitomycin C, Adriamycin, and Epirubicin(11). Urethral UC with prostate stroma invasion and in BCG-unresponsive patients require a radical radical cystectomy with lymphadenectomy $(20)$. Neoadjuvant platinum-based chemotherapy could be done in patients with advanced disease. Prostatic stromal invasion had a much higher LN metastasis rate compared with patients without stromal invasion. Lymphatic drainage from the prostate usually occurs to internal and external iliac regions, the obturator region, and the presacral region. Pararectal, paravesical and inguinal regions are considered to be aberrant lymph node regions. The reported incidence of lymph node metastases from prostate cancer to pararectal LN is 3\%(7), being a rare case. In prostate cancer, Gleason score was significantly correlated to the presence of positive lymph nodes in the pararectal region(15) indicating that highly malignant tumors tend to metastasize to pararectal LNs. The first patient in this report had prostate stroma invasion and metastasis to pelvic LN(obturator and pararectal). Obturator LN metastasis did not increase in size and pararectal metastasis was growing gradually. Differential growth of LN metastasis might represent heterogeneity of the tumor. LN metastasis is an independent prognostic factor for worse OS in patients with PU recurrence. The only curative treatment is the radical cystoprostatectomy with pelvic lymphadenectomy with or without neoadjuvant platinum-based chemotherapy $(9)$.

\section{Conclusion}

The true incidence of PU recurrences might be underestimated. These recurrences are often diagnosed incidentally. Patients successfully treated for high-risk multiple bladder cancers are at increased risk for PU recurrence. They are candidates for more frequent and attentive follow-up by endoscopy, urinary cytology, and CT scan and need aggressive treatment for their recurrences to prevent invasion into the prostate and further lymphatic metastasis.

\section{Conflict of Interest}

The authors have no conflict of interest in the subject matter or materials discussed in this manuscript.

\section{Author contribution}

SH and VB made substantial contributions to the conception, acquisition of data; VB conceived of the study, reviewed the literature, analyzed and interpreted the data, drafted and revised the manuscript. All authors read and approved the final version of the manuscript.

\section{Consent for publication}

Written informed consent was obtained from the patients and their relatives for publication of this case report and any accompanying images. A copy of the written consent is available for review by the Editor in Chief of this journal upon request.

\section{Figure legends}

Figure 1. The urethral tumor was resected by TUR and a deep invasion into the prostate was revealed (A). The pathological diagnosis was UC with prostate stromal invasion (B, Hematoxylin-Eosin Stain, 200x). One month after the operation recurrent urethral tumor was found during cystoscopy (C). CT revealed obturatory LN (D) and pararectal LN (E) metastasis which gradually enlarged (F).

Figure 2. Initial CT revealed multiple papillary bladder tumors (A). Urethral tumor at the verumontanum (B) was resected (C) and no residual tumor was present (D). Papillary UC without prostate tissue invasion (E, Hematoxylin-Eosin Stain, 200x). No recurrence was detected during follow up cystoscopy (F).

\section{References}


1. Bates, HR, Jr. 1969. Transitional cell carcinoma of the prostate. J Urol. 101 (2): 206-207.

2. Calderon Cortez, JF, Territo, A, Fontana, M, et al. 2021. Primary urethral carcinoma: Results from a single center experience. Actas Urol Esp 21 (S0210-4806(21)00096-6.).

3. Freeman, JA, Esrig, D, Stein, JP, and Skinner, DG. 1994. Management of the patient with bladder cancer. Urethral recurrence. Urol Clin North Am. 21 (4): 645-651.

4. Gakis, G, Black, PC, Bochner, BH, et al. 2017. Systematic Review on the Fate of the Remnant Urothelium after Radical Cystectomy. Eur Urol 71 (4): 545-557.

5. Herr, HW. 1998. Extravesical tumor relapse in patients with superficial bladder tumors. J Clin Oncol. $16(3): 1099-1102$.

6. Herr, HW and Donat, SM. 1999. Prostatic tumor relapse in patients with superficial bladder tumors: 15-year outcome. J Urol.161 (6): 1854-1857.

7. Holl, G, Dorn, R, Wengenmair, H, Weckermann, D, and Sciuk, J. 2009. Validation of sentinel lymph node dissection in prostate cancer: experience in more than 2,000 patients. Eur J Nucl Med Mol Imaging. 36 (9): 1377-1382. doi: 1310.1007/s00259-00009-01157-00252. Epub 02009 May 00259.

8. Ichihara, K, Kitamura, H, Masumori, N, Fukuta, F, and Tsukamoto, T. 2013. Transurethral prostate biopsy before radical cystectomy remains clinically relevant for decision-making on urethrectomy in patients with bladder cancer. Int J Clin Oncol 18 (1): 75-80.

9. Janisch, F, Abufaraj, M, Fajkovic, H, et al. 2019. Current Disease Management of Primary Urethral Carcinoma. Eur Urol Focus5 (5): 722-734.

10. Kirkali, Z and Canda, AE. 2006. Superficial urothelial cancer in the prostatic urethra. ScientificWorldJournal 6 (2603-2610.

11. Kirkali, Z and Canda, AE. 2006. Superficial Urothelial Cancer in the Prostatic Urethra. The Scientific World Journal 6 (Article ID 189746.

12. Liedberg, F, Chebil, G, and Mansson, W. 2007. Urothelial carcinoma in the prostatic urethra and prostate: current controversies. Expert Rev Anticancer Ther 7 (3): 383-390.

13. Matzkin, H, Soloway, MS, and Hardeman, S. 1991. Transitional cell carcinoma of the prostate. J Urol. 146 (5): 1207-1212.

14. Mazzucchelli, R, Barbisan, F, Santinelli, A, et al. 2009. Prediction of prostatic involvement by urothelial carcinoma in radical cystoprostatectomy for bladder cancer. Urology 74 (2):385-390.

15. Meijer, HJ, Fortuin, AS, van Lin, EN, et al. 1016. Geographical distribution of lymph node metastases on MR lymphography in prostate cancer patients. Radiother Oncol 106 (1): 59-63.

16. Mungan, MU, Canda, AE, Tuzel, E, Yorukoglu, K, and Kirkali, Z. 2005. Risk factors for mucosal prostatic urethral involvement in superficial transitional cell carcinoma of the bladder. Eur Urol 48 (5): 760-763.

17. Nixon, RG, Chang, SS, Lafleur, BJ, Smith, JJ, and Cookson, MS. 2002. Carcinoma in situ and tumor multifocality predict the risk of prostatic urethral involvement at radical cystectomy in men with transitional cell carcinoma of the bladder. J Urol 167 (2 Pt 1): 502-505.

18. Pagano, F, Bassi, P, Ferrante, GL, et al. 1996. Is stage pT4a (D1) reliable in assessing transitional cell carcinoma involvement of the prostate in patients with a concurrent bladder cancer? A necessary distinction for contiguous or noncontiguous involvement. J Urol155 (1): 244-247.

19. Pichler, R, Tulchiner, G, Oberaigner, W, et al. 2017. Effect of Urinary Cytology for Detecting Recurrence in Remnant Urothelium After Radical Cystectomy: Insights From a 10-year Cytology Database. Clin 
Genitourin Cancer 15 (5): e783-e791.

20. Walsh, DL and Chang, SS. 2009. Dilemmas in the treatment of urothelial cancers of the prostate. Urol Oncol. 27 (4):352-357.

21. Wishnow, KI and Ro, JY. 1988. Importance of early treatment of transitional cell carcinoma of prostatic ducts. Urology32 (1): 11-12.

22. Zhang, S and Guo, Z. 2018. Case report of a primary prostatic urothelial carcinoma patient with sustained fever. OncoTargets and Therapy 11 (4547-4550.

23. Zhou, J, Yang, C, Lu, Z, et al. 2019. Primary urothelial carcinoma of the prostate: A rare case report. Medicine (Baltimore) 98 (3): e14155. 

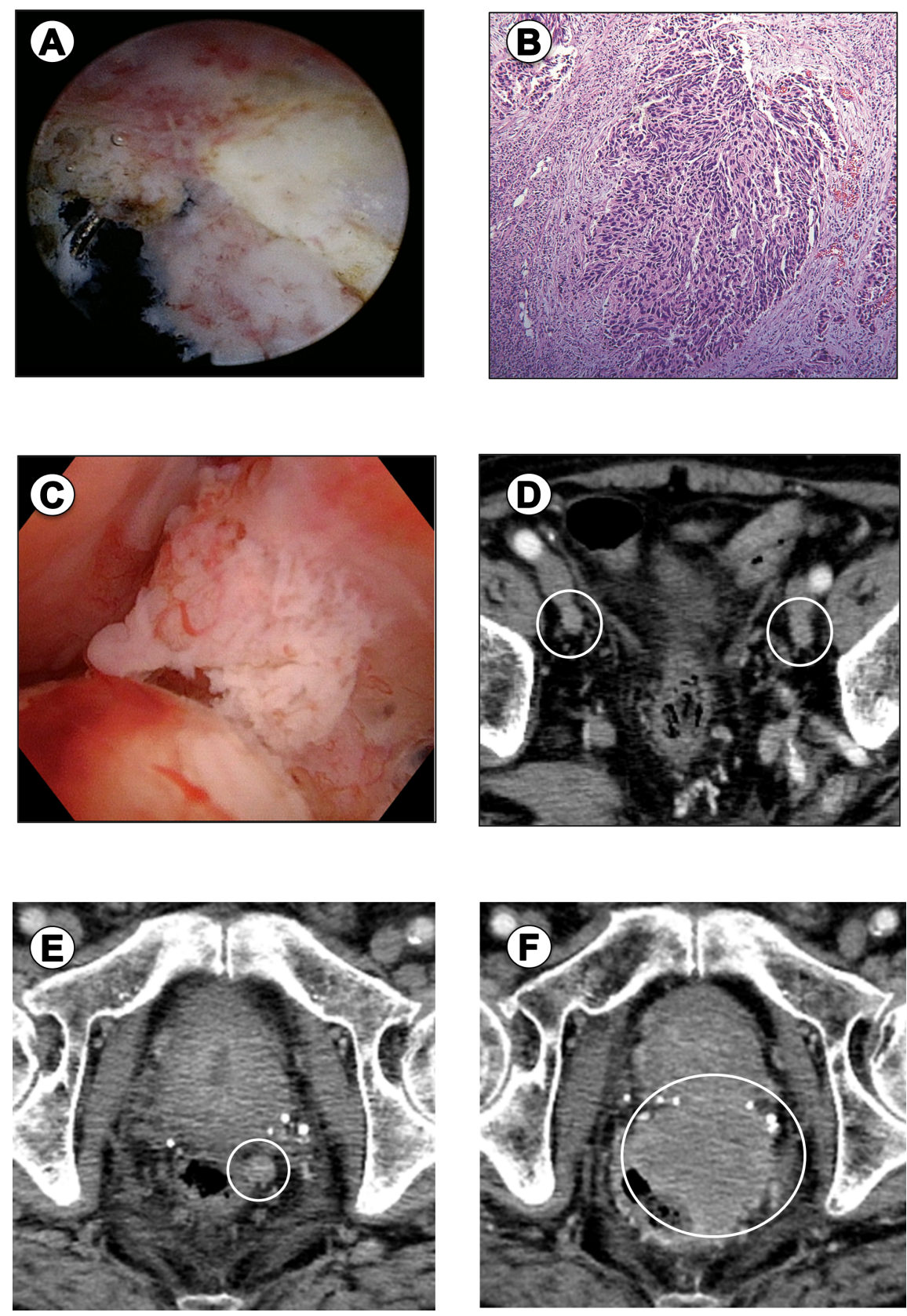

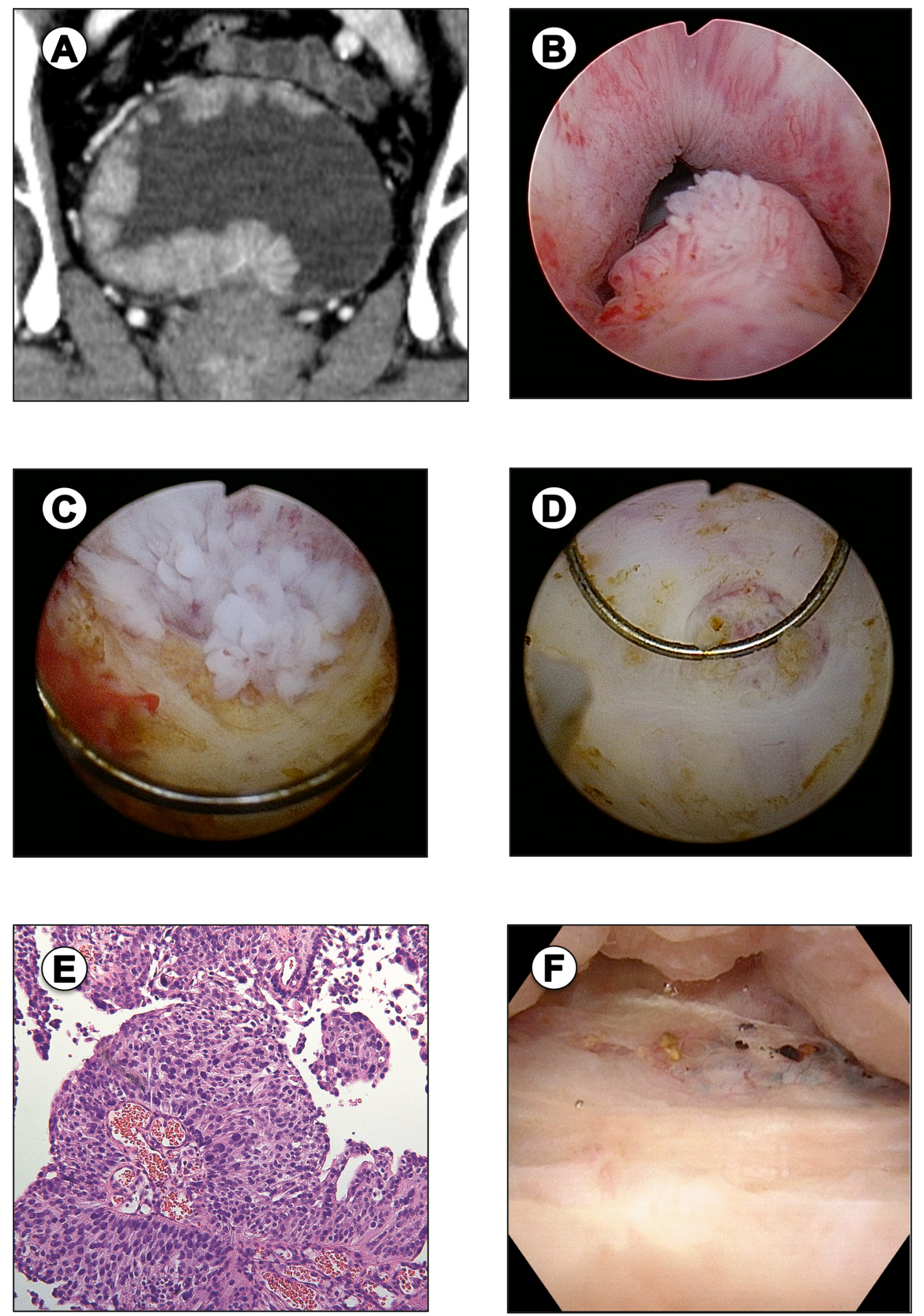